\title{
UtLlizAÇ̄o de recursos E Custos eM OSTEOPOROSE
}

\author{
*S.C. Kowalski, V.L. Suenzfeld, M.B. Ferraz
}

Disciplinade Reumatologiada UniversidadeFederaldeSãoPaulo-Escola PaulistadeMedicina,S.Paulo,SP

RESUMO - A osteoporose é uma doença caracterizada por baixa massa óssea e deterioraçäo da microarquitetura do tecido ósseo, com conseqüente aumento da fragilidade óssea e suscetibilidade a fraturas. Os recursos utilizados no tratamento de fraturas por osteoporose são siginificativos e com custos elevados.

OBßETTO. Dimensionar a utilização de recursos e custo anual por pacientes com osteoporose pós-menopausa.

Métodos. Cem pacientes foram consecutivamente selecionados do ambulatório de doenças osteometabólicas da Universidade Federal de São Paulo-Escola Paulista de Medicina (UNIFESPEPM), entre abril de 1997 a agosto de 1998. Os critérios de inclusão foram: osteoporose pós-menopausa (OMS, 1994) há pelo menos um ano; mínimo de um ano em acompanhamento ambulatorial; mínimas condiçöes de entendimento e expressão verbal para responder aos questionários. Características socioeconômicas, clínicas, utilização de recursos e custos no último ano foram levantadas através de entrevistas empregando-se dois questionários. Os custos unitários dos recursos utilizados no Serviço Público de Assistência à Saúde basearam-se na Tabela SUS de agosto de 1998.

ResultadOS. A média de idade foi 65,85 anos e a renda familiar média-mensal, R\$534,14. Foram realizadas em média sete consultas/paciente/ano. Das pacientes, $77 \%$ usaram cálcio e $38 \%$ estrógenos por algum período durante 0 último ano. Os custos médios totais anuais para 0 tratamento das pacientes com osteoporose pós-menopausa, sob a perspectiva da sociedade, no Sistema Público em São Paulo, foram de R\$ 908,18/paciente/ano.

Conclusáo. Os custos com o tratamento de osteoporose pagos pelas pacientes representaram $11 \%$ da renda familiar mensal média (R\$534). Em função do envelhecimento da população e aumento da incidência de osteoporose, políticas de alocação racional de recursos basedas em análises econômicas devem ser implementadas.

UnITERMOS: Osteoporose. Custode tratamento. Gestãoderecursos.

\section{INTRODUÇÃo}

Aosteoporose éuma doença caracterizada por baixa massa óssea e deterioração da microarquitetura do tecido ósseo, com conseqüente aumento da fragilidade ósseae suscetibilidadeàfraturas I

Komatsu, em Marília-SP, avaliou a incidência de fratura de quadril. Entremulheres de 50 a 59 anos foi de 27,8 casos, e entre 60 a 69 anos, 150,8 por 100.000 habitantes porano?

Osrecursos utilizados notratamento de fraturas por osteoporose representaram, em 1995 nos EUA, um total de 432 mil

*Correspondência:

Rua Botucatu $7403^{\circ}$ andar CEP 04023062 São

Paulo SP Brasil - telefone/fax 0xx 11 5579-6665 email sergiokowalski@ig.com.br hospitalizações, aproximadamente $2,5 \mathrm{mi}-$ Ihões de consultas médicase 180 mil admissões em Casas de Repouso ${ }^{3}$.

NoBrasil, não existem dados relacionadosàutilização de recursos e custos decorrentes da osteoporose pós-menopausa. Consequentemente, o presente trabalho procuroulevantar esses dados em um hospital universitário de atendimento terciário do Serviço Público de Assistênciaà Saúde.

\section{Métodos}

Cem pacientes foram selecionadas, consecutivamente, no ambulatório de Osteoporose daUNIFESP-EPMentreabril de 1997 a agosto de 1998.

Os critérios de inclusão foram: mulheres com diagnóstico de osteoporose pós- menopausa (OMS, 1994) 4 há pelo menos umano; pelomenos um ano deacompanhamento ambulatorial retroativo à data da entrevista; consentimento verbal de participação no estudo; mínimas condições de entendimentoeexpressãoverbal para responder aos questionários. Critérios de exclusão: doenças potencialmente causadoras de osteoporose secundária, acidentevascular cerebral, demência, litíase renal, uso de dilantina, fenobarbital, corticóides, heparina, imunossupressores; ausência de prontuário médico.

Os dadosforam colhidos em entrevistas individuais, apartirdoemprego de três questionários. O questionário I foi utilizado na coleta de dados de identificação, sociodemográficos, econômicose clínicos, perda de 
dias de trabalho e/ou aula em função de cuidados com a saúde nos últimos 12 meses (inclusive dos acompanhantes).

Dentre os recursos utilizados com o tratamento da osteoporose, na UNIFESPEPM ou outro serviço, investigou-se principalmente o número de consultas, internações, cirurgias, exames complementares solicitados, medicação utilizada e tratamentos alternativos. Foi levantada também a utilização de órteses e equipamentos auxiliares na locomoção, meios de transportes, contratação deserviços, além deadaptações no trabalho e na residência das pacientes; atividades domésticas não realizadasemfunção do estado de saúde e contratação de prestadores desses serviços. Questionouse igualmente os recursos utilizados com doenças associadas, considerando-se os regulares e os eventuais, como consultas de emergência e exames esporádicos.

Oquestionário 2(SF-36)foiutilizadopara avaliação de qualidade de vida das pacientes. Sua escala varia de 0 (pior resultado) a 100 (melhor resultado) ${ }^{5}$. Oquestionário 3 serviu para complementar informações referentes às características clínicas e recursos médicohospitalares (ex.: examescomplementares, medicação). Os dados foram extraídos do prontuário médico daUNIFESP-EPM.

Atodosos recursos utilizados foram atribuídos custos em reais ( $R \$$ ) referentes a agosto de 1998 (IR $\$=$ US\$0.85). Os resultados foram obtidos a partir do produto dos custosunitários de cadarecurso pelo número de vezes que foram utilizados nos últimos doze meses. O produto foi dividido por 100 (número de pacientes), chegando-seao customédio por paciente/ano. Os custos totais resultaram da soma das médias dos custos diretos e indiretos. Todos os custos foram apresentadossobaperspectivadasociedade, ouseja, todos aqueles pagos, independente de quem tenhasido o pagador.

Custos diretos - Foram considerados aqueles relacionadosàs consultas, exames, tratamentos, cirurgias, internações, utilização de órteses, equipamentos, adaptações no domicílio e/ou trabalho e transportes. Custos com contratação de prestadores de serviços em decorrência de algumalimitação apresentada pelas pacientes no último ano também foram estimados. Os custos com medicamentos foram calculados a partir da Tabela ABCFARMA (agosto/I 998). Para o etidronato dissódico, calciferol, carbonatode cálcioe cartilagem de tubarão osvaloresforam baseadosemumafarmácia demanipulação da cidade de São Paulo. Os custos unitários dos recursos médicos utilizados basearam-sena Tabela SUS de agosto de 1998. Para os meios de transporte foi utilizada tabela da SecretariaEstadual de Transportes.

Custos indiretos - Referiram-seàs perdas de dias de trabalho das pacientes e/ou acompanhantes em função dos cuidados com saúde. Dividiu-se o salário mensal de cada paciente e/ou acompanhante por 30 dias, multiplicou-se oresultado pelo número dedias de trabalho perdidos de cada paciente e/ou acompanhante. Dividiu-se o produto pelo número de pacientes $(100)$ e obtevese os custos indiretos médios.

AnáliseEstatística-Utilizamos aanáliseestatística descritiva (média e desvio-padrão) paraacaracterizaçãodemográficae clínica das pacientes estudadas. Os recursos utilizadose seus respectivos custosforam apresentados através de média por paciente por ano.

Aspectos Éticos-Otrabalhofoianalisado e aprovado pela Comissão de Ética da UNIFESP-EPM. Todas as pacientes consentiram em participar do estudo após apresentação dos objetivose garantia da manutenção da confidencialidade das informações.

\section{RESULTADOS}

Foram analisadas 100 mulheres com osteoporose pós-menopausa. Amaior parte das pacientes eram brancas ( $95 \%)$ e a idade média foi 65,85 anos (4I - 84). A renda mensal familiar média foi de $\mathrm{R} \$$ 535,00 , sendo que $43 \%$ tinham rendafamiliar entre I e 3 salários mínimos ( $R \$ 120,00$ - 360,00), 28\% entre 3 e 5 salários míni$\operatorname{mos}(R \$ 360,00$ - $R \$ 600,00)$ e $29 \%$ mais de 5 salários mínimos (>R $\$ 600,00) .79 \%$ das pacientesnão trabalhavam no período de realização do estudo(Tabela I).

Quarentaedois por cento das pacientes já haviam sofrido ao menos uma fratura relacionada à osteoporose. Fraturas de fêmur, vértebras e punho totalizaram $30 \mathrm{ea}$ soma de todas as outras fraturas chegoua 25 (Tabela 2).

Em relação ao questionário SF-36, a maioria das pacientes situou-se em valores intermediários, sendo os aspectosfísicos os menores $(49,64)$ e aspectos sociais os melhores $(67,39)$. Quase todas as pacientes $(96 \%)$ tinham ao menos uma doença associada, sendo mais comuns a artrose (44\%), hipertensão arterial sistêmica (40\%) efibromialgia (37\%).

As consultas médicas para o tratamento de osteoporose (327) representaram $42 \%$ do total de consultas médicas. Os exames complementares mais solicitados estavam relacionados com o metabolismo ósseo. A média de solicitações de densitometria ósseafoi de 0,85 por paciente por ano. Observou-se um alto consumo de cálcio (77\%)e vitamina $D(47 \%)$; os estrógenos foram utilizados por menos da metade das pacientes $(38 \%)$. $15 \%$ das pacientes utilizaram cartilagem de tubarão, valor superiorà utilização de alendronato $(12 \%)$, etidronato (10\%) e flúor (11\%). Os medicamentos mais utilizados no tratamento das doenças associadas foram os analgésicos (54\%), diuréticos (36\%), antidepressivos (34\%).

Os custos médios com medicamentos para osteoporose representarama soma de $\mathrm{R} \$ 35 \mathrm{I}, 28 /$ paciente/ano e para as doenças associadas $\mathrm{R} \$ 217,31 /$ paciente/ano. Notamos que o tratamento da osteoporose foi responsável por $61 \%$ dos custos com me- 


\begin{tabular}{|c|c|c|c|c|}
\hline \multicolumn{3}{|c|}{$\begin{array}{c}\text { Tabela I - Dados demográficos e socioeconômicos das } 100 \text { pacientes com } \\
\text { osteoporose pós-menopausa atendidas no ambulatório de Reumatologia da } \\
\text { UNIFESP/EPM no período de um ano }\end{array}$} & \multirow{2}{*}{\multicolumn{2}{|c|}{$\begin{array}{l}\text { Tabela } 2 \text { - Características clínicas das } \\
100 \text { pacientes com osteoporose } \\
\text { pós-menopausa atendidas no } \\
\text { ambulatório de Reumatologia - } \\
\text { UNIFESP/EPM no período de um ano }\end{array}$}} \\
\hline $\begin{array}{l}\text { Idade } \\
\text { Média(dp) }\end{array}$ & & $65,85(8,82)$ & & \\
\hline Cor & $\begin{array}{l}\text { Branca } \\
\text { nãobranca }\end{array}$ & $\begin{array}{c}95 \\
5\end{array}$ & $\begin{array}{l}\text { Tempodediagnóstico,(anos) } \\
\text { Osteoporose }\end{array}$ & $4,4 \mid(3,27)$ \\
\hline Municípioderesidência & $\begin{array}{l}\text { SãoPaulo } \\
\text { Outros }\end{array}$ & $\begin{array}{l}87 \\
13\end{array}$ & média(dp) & \\
\hline Estadocivil & $\begin{array}{l}\text { Casada } \\
\text { Viúna } \\
\text { Solteira } \\
\text { Divorciada }\end{array}$ & $\begin{array}{l}49 \\
33 \\
15 \\
3\end{array}$ & $\begin{array}{l}\text { Tempodemenopausa, (anos) } \\
\text { média(dp) } \\
\text { Fraturs* }\left(n^{9}\right)\end{array}$ & $18,79(10,22)$ \\
\hline $\begin{array}{l}\text { Anosdeestudo } \\
\text { média(dp) }\end{array}$ & & $3,73(3,56)$ & $\begin{array}{l}\text { QuestionárioSF36 } \\
\text { média(dp) }\end{array}$ & \\
\hline $\begin{array}{l}\text { Rendafamiliarmensal(R\$) } \\
\text { média(dp) }\end{array}$ & & $534,14(448,38)$ & $\begin{array}{l}\text { meapaacip) } \\
\text { Aspectosfíficos }\end{array}$ & $\begin{array}{l}58,71(24,00) \\
49,64(40,04)\end{array}$ \\
\hline Moradia & $\begin{array}{l}\text { Própria } \\
\text { Alugada }\end{array}$ & $\begin{array}{l}88 \\
12\end{array}$ & $\begin{array}{l}\text { Dor } \\
\text { Estadogeral desaúde }\end{array}$ & $\begin{array}{l}58,01(22,80) \\
66,15(22,86)\end{array}$ \\
\hline Situação funcional & $\begin{array}{l}\text { Ativas } \\
\text { Inativas }\end{array}$ & $\begin{array}{l}21 \\
79\end{array}$ & $\begin{array}{l}\text { Vitalidade } \\
\text { Aspectossociais }\end{array}$ & $\begin{array}{l}53,21(22,36) \\
67,39(25,74)\end{array}$ \\
\hline Razãodeaposentadoria(\%) & $\begin{array}{l}\text { Idade } \\
\text { doença* } \\
\text { tempodeserviço }\end{array}$ & $\begin{array}{l}18 \\
14 \\
8\end{array}$ & $\begin{array}{l}\text { Aspectosemocionais } \\
\text { Saúdemental }\end{array}$ & $\begin{array}{l}51,90(42,71) \\
56,89(24,76)\end{array}$ \\
\hline $\begin{array}{l}\text { *4lombalgia, 2Hipertensão } \\
\text { I Cardiopatia; I DoençaNe }\end{array}$ & ca; 2Artrose, 2Oste & iquiátrica; & $\begin{array}{l}\text { *fraturas defêmur, vértebrasep } \\
\text { outrasfraturas }=25 \text {. }\end{array}$ & nho $=30$, \\
\hline
\end{tabular}

dicamentos para este grupo de pacientes estudadas. Os custos médios totais dos recursos médico-hospitalares foram de $\mathrm{R} \$$ $748,81 /$ paciente/ano, sendo a maior parcela relativa aos medicamentos e a menor a equipamentos auxiliares para a marcha (ex. órteses, bengalas) (Tabela 3).

Os custos médico-hospitalares representaram $83 \%$ dos custos diretos totais. Dentre os custos nãomédico-hospitalares, os meios de transporte responderam pela maior parcela ( $\mathrm{R} \$ 151,82 /$ paciente/ano).

Os custos indiretos ( $R \$ 155,82$ ) representaram perdas de dias trabalhados por pacientes eacompanhantes no período de umano.
Os custos médios totais pagos pelas pacientes foram de R\$ 730,78/paciente/ano.

ATabela4ilustra os custos totais (diretos eindiretos) paraa sociedade, no tratamento das $100 \mathrm{pa}$ cientes com osteoporoseatendidas no período de um ano. Ocustomédio anual por paciente foi de $\mathrm{R} \$ 908,19$.
Tabela 3 - Custos diretos totais dos recursos médico - hospitalares (consultas, exames complementares, cirurgias, medicamentos e equipamentos) utilizados pelas pacientes com osteoporose pós-menopausa atendidas no ambulatório de Reumatologia da UNIFESP/EPM no período de um ano ( $R \$$ ago 1998)

\begin{tabular}{lc}
\hline Recursos & Custos totais (R\$) \\
Cirurgias* & $2.150,05$ \\
Consultas* & $3.595,36$ \\
Examescomplementares* & $11.840,00$ \\
Medicamentos** & $56.860,09$ \\
Equipamentos & 436,00 \\
Total & $74.881,50$ \\
Média/pac/ano & 748,81 \\
*TabelaSUS-SistemaÚnicodeSaúdeAGO 1998 & \\
**TabelaABCFARMAAGO 1998 & \\
\hline
\end{tabular}




\section{Discussão}

Aosteoporose é uma doença que tem despertado grande interesseem Saúde Pública, pois com o crescimento e envelhecimento da população mundial, o número de pessoasidosas que se encontram na faixa de risco para fraturas e mesmo a incidência idade-específica estáaumentando consideravelmente. NoBrasil, estima-se quea proporção de idosos (maior de 65 anos) saltará de 5, $1 \%$ em 2000, para 14,2\% em 20506.

No presente trabalho, a média de idade das pacientes foi de 65,85 anos. Esse grupo poderiaestar enquadradonos $8,5 \%$ dapopulação da região metropolitana de São Paulo acima dos 65 anos de idade, no ano de 1995. Considerando-se esta previsão, com uma esperança devidade $16, \mathrm{I} I$ anos, poderiase estimar que as mulheres deste grupo sobreviveriam atéaidade de 82 anos, período em que as fraturas por osteoporose, principalmentedequadril, têmumaincidênciaaumentada de formaexponencial ${ }^{7}$.

Quase metade (42\%) das pacientes deste trabalho constituíam um grupo de risco para novas fraturas, ou seja, já haviam sofrido previamente algum tipo de fratura relacionadaà osteoporose ${ }^{8}$.

Umoutrofatoragravanteparaadebilidade física dessas pacientesé que $96 \%$ possuíam ao menos uma doença associada à osteoporose, sendo que $72 \%$ tinham duas ou mais doenças associadas; as mais freqüentes foram fibromialgia, hipertensão arterial e artrose. Esses resultados são semelhantes aosde umestudoempopulação domiciliar, na área metropolitana da região sudeste do Brasil, onde pelo menos $17 \%$ dos pacientes acima dos 60 anos pertencentes ao grupo commenor poder aquisitivo apresentavam, pelomenos, cinco doenças simultâneas 9

NoBrasil, umlevantamento constatouque a proporção de mulheres acima dos 65 anos quevivem sozinhas vemaumentando. Alcançou $14,9 \%$ em 1989 e dessas, $60 \%$ possu- íamrendainferior ou igualaumsaláriomínimo. Dentre as pacientes maiores de 70 anos, $17,6 \%$ moravamsozinhas ${ }^{10}$.

Caso sofressem algum tipo de fratura, as pacientes do estudo atual enfrentariam basicamente dois problemas. Primeiro, a Tabela 4 - Custos totais (diretos e indiretos), sob a perspectiva da sociedade, no tratamento das 100 pacientes com osteoporose pós-menopausa atendidas no ambulatório de Reumatologia/Osteoporose da UNIFESP/EPM no período de um ano. Valores de referência Tabela SUS 98 (R\$ ago 1998)

\begin{tabular}{lc}
\hline Custos para a sociedade & (R\$) \\
Diretos & $90.663,95$ \\
Indiretos & 155,82 \\
Total & $90.819,77$ \\
Média/pac/ano & 908,19 \\
\hline
\end{tabular}

precariedade de re-

cursos financeiros para seu tratamento (somente $21 \%$ tinham emprego remunerado e $43 \%$ tinham renda familiar mensal entre I e 3 salários mínimos). Segundo, teriam dificuldades para desempenhar as atividades da vida diária. Cooper relatou que um ano após a fratura de quadril, $40 \%$ das pacientes estudadas ainda não estariam aptas a caminhar sem auxílio e $60 \%$ teriam dificuldades em realizar ao menos uma atividade da vida diária como vestir-se, banhar-se ou preparar sua alimentação".

Verificou-se que $32 \%$ das pacientes no presente estudo apresentaramalguma limitação para realizar atividades domésticas, porém somente cinco contrataram servidoras comesta finalidade, a um custo médio mensal de $R \$ 50,00$. O custo deste serviço deve ter sido o motivo da baixa utilização deste tipo de recurso.

Em relação à qualidade de vida, todos os domínios do questionário SF-36 apresentaram valores baixos, situados ao redor da metade da escala de 0 a 100 , onde zero representa o pior resultado e 100 o melhor.

O número médio de consultas realizadas foi de sete por paciente ao ano, sendo três para tratamento da osteoporosee quatro para tratamento das doenças associadas.

Em relação ao número de consultas, os resultados do presente estudo estão próximos dos encontrados nos EUA entre 1984 e 1986, onde pacientes com doenças músculo-esqueléticas esem co-morbidadesrealizaram em média 4,5 consultas por ano. Aqueles com co-morbidades realizaram, em média, 9,6 consultas por ano ${ }^{12}$.

Os exames complementares mais realizados estavam relacionados com o metabolismo ósseo. A média de solicitações de densitometria ósseafoi de 0,85 por paciente por ano. Este valor é um pouco superior ao sugerido pelos Guidelines for Diagnosis and Management of Osteoporosis, ou seja, um exame a cada dois anos ${ }^{8}$. Um dos prováveis motivos para isto seria que a UNIFESP é um centro de pesquisa onde estudos com objetivos diversos ocorrem simultaneamente, resultando em repetição de exames. Além disso, a desorganização de alguns prontuários médicos levaà perda dos resultados, fazendo com que novos exames sejam realizados.

Uma grande parcela das pacientes (77\%) utilizou cálcio, $47 \%$ vitamina D e $38 \%$ estrógenos em algum período no ano do estudo, sendo toda a medicação comprada pelas próprias pacientes. O fato do cálcio ser a droga mais utilizada está de acordo com a tendência mundial, sendo esta a droga não-hormonal mais utilizada para o tratamento da osteoporose, e de grande aderência ${ }^{10}$. O custo mensal para o cálcio foi de R\$15,6 (US\$ 13,33) e R\$16,5 
(US\$ 14, I) paravitaminaD (emUS\$de 1998).

Os estrógenos são os medicamentos de maior custo/efetividade para osteoporose. Embora uma parcela considerável (38\%) os tenha utilizado, com um custo mensal de $R \$ 15,4$, sabe-se que $50 \%$ das pacientesabandonam o tratamento entre 6 e 12 meses após a prescriçãa ${ }^{4}$.

Oácidoacetil salicílico foi o medicamento mais empregado dentre as drogas paraas doenças associadas, seguido pelos diuréticos (36\%) e antidepressivos (34\%). 0 custo mensal do ácido acetil salicílico foi de $\mathrm{R} \$ 7,2$ (US\$ 6, I5) (em US\$ de 1998).

Vanjaarsveld etal., nos EUA, analisaram custos em pacientes com artrite reumatóide (em US\$ de 1997). Os valores mensais do cálcio, vitamina Deácido acetilsalićlico foram respectivamente US\$ 10,3; US\$9,33 eUS\$ $5,\left.8\right|^{13}$. Percebe-se que os medicamentos apresentam valores semelhantesaos do presente estudo. No entanto, temos de um lado um país desenvolvido com acesso ao sistema desaúde para umagrande parcela dapopulaçãoe, por outrolado, o Brasil, ondea maioria das pessoas tem dificuldade de acesso aos serviços deassistênciaàsaúdee baixasituação socioeconômica.

Um número representativo (15\%) das pacientes do estudo atualadmitiuter tomado, poralgum período, o produto cartilagem de tubarão. Essefato ésurpreendente, pois seu custo mensal foi aproximadamente $R \$ 33,6$. Drogascomevidênciascientíficas deefetividade deixaram de usadas, como o etidronato ( $R$ \$ 20,85/mês) e alendronato ( $R \$ 96,6 /$ mês). Emboraa cartilagem detubarãonão sejauma substância empregada naUNIFESP, pela ausênciadeevidênciasquecomprovemsuaeficácia/efetividade, énecessário fazerumlevantamentodas causas dessa utilização.

Dados referentesao levantamentodomiciliar do IBGE,em 1991, demonstraram quena regiãometropolitanadeSão Paulo, as despesas com remédios consumiram $4,61 \%$ da renda mensal nas famílias que recebiam de 2 a 3 salários mínimos(SM)e 3,96\% naquelas que percebiamentre 3 e $5 \mathrm{SM}^{14}$.

Considerando-seamédiamensal darenda familiar das pacientes do estudo atual de R\$ 534, I4(5SM), as despesasforamo dobroem relaçãoàpopulação domiciliardeSão Paulo,em 1991.As pacientes gastaram, em média, com drogas para osteoporose em torno de R\$ 29,27 por mês, $5 \%$ da renda familiar média. Somando-seo custo do tratamento das doenças associadas, chega-seaovalor de $\$ 47,38$ pormês, $9 \%$ darendafamiliarmédiacomprometidacommedicação.

Considerando-se o contexto socioeconômico da população brasileira, seria importante que, dentro das normas de aprovação de medicamentos, fossem criados estímulos para obter-se um custo compatível com nossa realidade.

No presente estudo, as despesas com tratamento, soba perspectiva das pacientes, foram de $\mathrm{R} \$ 730,78$ por paciente por ano ou $R \$ 60,89 /$ mês ( $11 \%$ da renda familiar mensal). Adistribuição foia seguinte: medicação $(77,8 \%)$, meios de transporte $(20,77 \%)$, contratação de serviços e equipamentos (I,3\%).

Considerando-se os custos diretos médios anuais sob a perspectiva da sociedade, chegou-se ao valor de $\mathrm{R} \$ \mathbf{9 0 6 , 6 3}$ por paciente, sendo $82 \%$ utilizados com recursos médico-hospitalares e 18\% com recursos não médico-hospitalares. Percebe-se que os itens de maior peso nos custos do tratamento da osteoporose recaem sobre os pacientes que devem comprar os medicamentos e pagar as tarifas de transporte, tudo a partir de uma renda familiar médiade $\mathrm{R} \$ 534,00$.

Acrescentando-se os custos indiretos médios anuais de R\$ I,55 por paciente, chegamos aos custos médios totais de R\$ 908, I 8 por paciente por ano.

Nosso estudo é pioneiro no país e foi retrospectivo, por esses motivos pode conter algumas imperfeições, principalmente por usar como fontes de informação as pacientes que eventualmente poderiam ter esquecido alguns itens referentes ao tratamento, e os prontuários que não registram todos os dados adequadamente. Damesma forma, devido ao seu curto período de realização, não houve casos de fraturas e internações em unidades de terapia intensiva, para comparar-se com estudos da literatura internacional.

No Brasil, trabalhos para determinara incidência e fatores de risco para osteoporose em nível populacional precisam ser elaborados. Estudos decusto-efetividadeno tratamento de osteoporose, utilizando-se métodos da Medicina Baseada em Evidências e análise econômica em saúde, podem apresentar resultados sobre os custos poupados com o emprego de diferentes intervenções. Paralelamente, deve-se analisaro grau de atenção com a osteoporose, por parte dos médicos, nos centros de atendimentoà saúde dos primários aos terciários.

Baseados nos resultados, propostas de intervenções para osteoporose que proporcionem os melhores resultados para 0 maior número possível de pessoas, com o menor custo, devem partir de políticas de saúde que envolvama participação de autoridades dasáreas médica, econômica, social e representantes da população.

\section{SUMMARY}

\section{RESOURCE UTILIZATION AND COSTS IN OSTEOPOROSIS}

BACKGROUND. Osteoporosis is characterized by low bone mass, deterioration of the microarchitecture of the bone tissueand increase susceptibility to fractures. Clinical manifestations are fractures and their complications. The most common fractures are hip,spine and wrist, although any bone is susceptible.

OBJECTIVES. Toassess the resource utilization and the annual costs incurred by 
patients with postmenopausal osteoporosis

METHODS. One hundred consecutive patients were studied in the Universidade Federal de São Paulo. The inclusion criteria were: postmenopausal osteoporosis (WHO 1994) forat least oneyearb) at least oneyearattendingtheoutpatient clinic, minimal comprehensive and speaking conditions. The socioeconomic and clinical characteristics, the resource utilization and costs in the last year were assessed using 2 questionnaires. The unitary costs were based in the Public Healthcare System Index (1998).

RESULTS. Themeanagewas66yearsandthe monthly family income was $R \$ 534.14$ (US\$ 456-in 1998). There was a mean of 7 visits/ patient/year. $77 \%$ of the patients used calcium and $38 \%$ used estrogens. The mean total annual costsforosteoporosistreatment, considering societal perspective, were $R \$ 908.18$ (US\$776-in 1998) per patient peryear.

CONCLUSION. The costs related to osteoporosis treatmentrepresented II\% of the mean monthly household income from the patients' perspective ( $R \$ 534=U S \$ 456)$. As the population is ageing and the osteoporosisincidence is increasing, health policy should be implemented to rationallyallocate the scarce resources available, based on economic analysis. [Rev Ass Med Brasil 200I; 47(3): 352-7]

\section{KeYwORDs: Osteoporosis. Costs. Resources} utilization.

\section{REFERÊNCIAS}

I. PeckWA. Consensus development conference: diagnosis, prophylaxis, and treatment of osteoporosis. Am J Med 1993; 94:646-50.

2. Komatsu E. Incidência de fraturas de quadril em Marília-SP [dissertação]. São Paulo: Universidade Federal de São Paulo - Escola Paulista de Medicina; 1998.

3. National Osteoporosis Foundation. Osteoporosis: review of the evidence for prevention, diagnosis and treatment and cost-effective analysis. Osteoporos Int 1998; (Suppl 4):SI-S6.

4. World Health Oraganization Study Group. Assessment of fracture risk and its application in screening for postmenopausal osteoporosis. Geneva: World Health Organization 1994. (WHO Technical Report Series 843)

5. Ciconelli RM. Tradução para a língua portuguesa e validação do questionário genérico de avaliação de qualidade de vida. SF-36 (BRASIL SF36). Rev Bras Reumatol 1999; 39: $143-50$.

6. Machado CC. Projeções multirregionais da população:o caso brasileiro(1980-2020) [tese]. Belo Horizonte: Universidade Federal de Minas Gerais; 1993.

7. Bagur A, Mautalen C, Rubin Z. Epidemiology of hip fractures in an urban population of Central Argentina. Osteoporos Int 1994; 4:332-5.

8. Janis JA, Delmas P, Burckhardt P, Cooper C, Torgerson D. Guidelines for diagnosis and management of osteoporosis. Osteoporos Int 1997; 7:390-406.

9. Ramos LR, Rosa TEC, Oliveira ZM, Medina MCG, Santos FR. Perfil do idoso em área metropolitana na região sudeste do Brasil: resultados de inquérito domiciliar. Rev Saúde Pública 1993, 27:87-94.

10. BerquóE. Características demográficas e sócioeconômicas da população idosa. Diferenças inter e intra-regionais. In: Seminário Nacional dos Especialistas Multidisciplinares em terceiraidade. Belo Horizonte. Anais. Belo Horizonte: Fundação João Pinheiro, 1992. p.4I-62.

I I. Cooper C. The crippling consequences of fractures and their impact on quality of life. Am J Med. 1997; 103 (Suppl 2 A): I2S-9S.

12. Felts W, Yelin E. The economic impact of the rheumatic diseases in the United States. J Rheumatol 1989; 16:867-884.

I 3. Van Jaarsveld CHM, Jacobs JWG, Schrijvers AJP. Direct cost of rheumatoid arthritis during the first six years:a cost-of-ilness study. Br J Rheumatol 1998; 37:837-47.

I4. Fundação IBGE. Pesquisa de orçamentos familiares. [online]. Disponivel em: URL: http://www.ibge.gov.br/pnad.

Artigo recebido: I I/05/200| Aceito para publicação: |3/08/200| 